\title{
GLOBAL DYNAMICS OF SOME FRACTIONAL DIFFERENCE EQUATIONS
}

\author{
H. EL-METWALLY ${ }^{1}$, E. M. ELABBASY ${ }^{1}$ \\ and M. AL-KAFF ${ }^{2}$ \\ ${ }^{1}$ Department of Mathematics \\ Faculty of Science \\ Mansoura University \\ Mansoura 35516 \\ Egypt \\ e-mail: eaash69@yahoo.com \\ emelabbasy@mans.edu.eg \\ 2Department of Mathematics \\ Hadhramout University \\ Yemen \\ e-mail: abohassien246@gmail.com
}

\begin{abstract}
We investigate the global dynamics of the equation

$$
x_{n+1}=\frac{x_{n-1}}{\alpha x_{n}^{2}+\beta x_{n} x_{n-1}+\gamma x_{n-1}}, \quad n=0,1, \ldots,
$$

where the parameters $\alpha, \beta$, and $\gamma$ are nonnegative numbers with

$\alpha+\beta+\gamma>0$ and the initial conditions $x_{-1}$ and $x_{0}$ are arbitrary positive real

numbers. The equilibrium points of the considered equation are obtained and
\end{abstract}

2010 Mathematics Subject Classification: 39A10.

Keywords and phrases: asymptotic stability, global convergence, periodic solution, local stability, rational difference equation.

Received November 29, 2018; Revised February 09, 2019

(C) 2018 Scientific Advances Publishers 
classified whether stable or unstable. The boundedness, the global stability and the periodicity of the solutions are investigated. Some numerical examples are given to illustrate the obtained results.

\section{Introduction}

We investigate the global behaviour of the equation

$$
x_{n+1}=\frac{x_{n-1}}{\alpha x_{n}^{2}+\beta x_{n} x_{n-1}+\gamma x_{n-1}}, \quad n=0,1, \ldots
$$

where the parameters $\alpha, \beta$, and $\gamma$ are nonnegative numbers with $\alpha+\beta+\gamma>0$ and the initial conditions $x_{-1}$ and $x_{0}$ are arbitrary positive real numbers.

Many authors and researchers are interesting in studying the global attractivity, the boundedness character and the periodic nature of nonlinear difference equations.

Hrustić et al. [13] studied the global dynamics and the bifurcations of a certain second-order rational difference equation with quadratic terms

$$
x_{n+1}=\frac{x_{n-1}}{a x_{n}^{2}+e x_{n-1}+f} .
$$

Kulenović et al. [17] investigate the global asymptotic stability and Naimark-Sacker bifurcation of the difference equation

$$
x_{n+1}=\frac{F}{b x_{n-1} x_{n}+c x_{n-1}^{2}+f} .
$$

Kulenović et al. [16] investigated the solutions of the difference equation

$$
x_{n+1}=\frac{\gamma x_{n-1}}{A+B x_{n}+C x_{n-1}} .
$$

Other related results on rational difference equations can be found in (([4]-[9]), [11], [14, 15], [18, 19], [21]). 
Let $I$ be some interval of real numbers and let

$$
f: I^{K+1} \rightarrow I
$$

be a continuously differentiable function. Then for every set of initial conditions $x_{-1}, x_{0} \in I$ the difference equation

$$
x_{n+1}=f\left(x_{n}, x_{n-1}, \ldots, x_{n-k}\right), \quad n=0,1, \ldots,
$$

has a unique solution $\left\{x_{n}\right\}_{n=-1}^{\infty}$. A solution of Equation (1.2) that is constant for all $n \geq-k$ is called an equilibrium solution of Equation (1.2). If

$$
x_{n}=\bar{x}, \text { for all } n \geq-k,
$$

is an equilibrium solution of Equation (1.2) then $\bar{x}$ is called an equilibrium point or simply an equilibrium of Equation (1.2).

Definition 1 (Permanence). Equation (1.2) is said to be permanent and bounded if there exist number $m$ and $M$ with $0<m<M<\infty$ such that for any initial condition $x_{-1}, x_{0} \in(0, \infty)$ there exists a positive integer $N$ which depends on these initial conditions such that $m<x_{n}<M$ for all $n \geq N$.

\section{Definition 2 [16] (Semicycles).}

(i) A positive semicycle of a solution $\left\{x_{n}\right\}$ of Equation (1.2) consists of a "string" of terms $\left\{x_{l}, x_{l+1}, \ldots, x_{\mu}\right\}$, all greater than or equal to the equilibrium $\bar{x}$ with $l \geq-1$ and $\mu \leq \infty$ and such that

$$
\text { either } l=-1, \quad \text { or } \quad l>-1 \quad \text { and } \quad x_{l-1}<\bar{x} \text {, }
$$

and

$$
\text { either } \mu=\infty \text {, or } \mu<\infty \text { and } x_{\mu+1}<\bar{x} \text {. }
$$


(ii) A negative semicycle of a solution $\left\{x_{n}\right\}$ of Equation (1.2) consists of a "string" of terms $\left\{x_{j}, x_{j+1}, \ldots, x_{\mu}\right\}$ all less than to the equilibrium $\bar{x}$ with $j \geq-1$ and $\mu \leq \infty$ and such that

$$
\text { either } j=-1, \quad \text { or } \quad j>-1 \quad \text { and } \quad x_{j-1} \geq \bar{x} \text {, }
$$

and

$$
\text { either } \mu=\infty \text {, or } \mu<\infty \text { and } x_{\mu+1} \geq \bar{x} \text {. }
$$

\section{Theorem A [1] (Linearized stability).}

Suppose that the function $F$ is continuously differentiable in some open neighbourhood of an equilibrium point $\bar{x}$. Let

$$
p_{i}=\frac{\partial f}{\partial v_{i}}(\bar{x}, \bar{x}, \ldots, \bar{x}), \quad \text { for } i=0,1, \ldots, k
$$

denote the partial derivative of $f\left(v_{0}, v_{1}, \ldots, v_{k}\right)$ with respect to $v_{i}$ evaluated at the equilibrium point $\bar{x}$ of Equation (1.1). Then the equation

$$
\gamma_{n+1}=p_{0} \gamma_{n}+p_{1} \gamma_{n-1}+\ldots+p_{k} \gamma_{n+k}, \quad n=0,1, \ldots,
$$

is called the linearized equation of Equation (1.1) about the equilibrium point $\bar{x}$ and the equation

$$
\lambda_{k+1}-p_{0} \lambda_{k}-\ldots-p_{k-1} \lambda-p_{k}=0,
$$

is called the characteristic equation of Equation (1.4) about $\bar{x}$.

Then the following statements are true:

(a) When all the roots of Equation (1.4) have absolute value less than one, then the equilibrium point $\bar{x}$ of Equation (1.2) is locally asymptotically stable.

(b) If at least one root of Equation (1.4) has absolute value greater than one, then the equilibrium point $\bar{x}$ of Equation (1.2) is unstable. 
The equilibrium point $\bar{x}$ of Equation (1.2) is called hyperbolic if no root of Equation (1.4) has absolute value equal to one. If there exists a root of Equation (1.4) with absolute value equal to one then the equilibrium $\bar{x}$ is called nonhyperbolic.

An equilibrium point $\bar{x}$ of Equation (1.2) is called a saddle point if it is hyperbolic and if there exists a root of Equation (1.4) with absolute value less than one and another root of Equation (1.4) with absolute value greater than one.

An equilibrium point $\bar{x}$ of Equation (1.2) is called a repeller if all roots of Equation (1.4) have absolute value greater than one.

Theorem B ([16]). Let $[a, b]$ be an interval of real numbers and assume that

$$
f:[a, b]^{2} \rightarrow[a, b]
$$

is a continuous function satisfying the following properties:

(a) $f(x, y)$ is non-increasing in $x \in[a, b]$ for each $y \in[a, b]$ and is non-decreasing in $y \in[a, b]$ for each $x \in[a, b]$.

(b) The difference equation Equation (1.1) has no solutions of prime period two in $[a, b]$.

Then Equation (1.1) has a unique equilibrium $\bar{x} \in[a, b]$ and every solution of Equation (1.1) converges to $\bar{x}$.

Theorem C ([22]). Assume that $f \in C[(0, \infty) \times(0, \infty),(0, \infty)]$ is such that $f(u, v)$ is decreasing in $u$ for each fixed $v$ and $f(u, v)$ is increasing in $y$ for each fixed $u$. Let $\bar{x}$ be a positive equilibrium of Equation (1.2). Then except possibly for the first semicycle every solution of Equation (1.2) has semicycles of length one. 
Theorem $\mathbf{D}$ ([12]). Let $\boldsymbol{J}$ be some interval of real numbers $f \in C\left[\boldsymbol{J}^{v+1}, \boldsymbol{J}\right]$, and let $\left\{x_{n}\right\}_{n=-v}^{\infty}$ be a bounded solution of the difference equation

$$
x_{n+1}=f\left(x_{n}, x_{n-1}, \ldots, x_{n-v}\right), \quad n=0,1, \ldots,
$$

with

$$
\boldsymbol{I}=\lim _{n \rightarrow \infty} \inf x_{n}, \quad \boldsymbol{S}=\lim _{n \rightarrow \infty} \sup x_{n} \quad \text { and with } \quad \boldsymbol{I}, \boldsymbol{S} \in \boldsymbol{J} .
$$

Then there exist two solutions $\left\{\boldsymbol{I}_{n}\right\}_{n=-\infty}^{\infty}$ and $\left\{\boldsymbol{S}_{n}\right\}_{n=-\infty}^{\infty}$ of Equation (1.5) with

$$
\boldsymbol{I}_{0}=\boldsymbol{I}, \quad \boldsymbol{S}_{0}=\boldsymbol{S}, \quad \boldsymbol{I}_{n}, \boldsymbol{S}_{n} \in[\boldsymbol{I}, \boldsymbol{S}] \quad \text { for all } n \in \mathbb{Z}
$$

and such that for every $N \in \mathbb{Z}, \boldsymbol{I}_{N}$ and $\boldsymbol{S}_{N}$ are limit points of $\left\{x_{n}\right\}_{n=-v}^{\infty}$.

Furthermore for every $m \leq-v$, there exist two subsequences $\left\{x_{r_{n}}\right\}$ and $\left\{x_{l_{n}}\right\}$ of the solution $\left\{x_{n}\right\}_{n=-v}^{\infty}$ such that the following are true:

$$
\lim _{n \rightarrow \infty} x_{r_{n}+N}=\boldsymbol{I}_{N} \quad \text { and } \quad \lim _{n \rightarrow \infty} x_{l_{n}+N}=\boldsymbol{S}_{N} \quad \text { for every } \quad N \geq m .
$$

The solutions $\left\{\boldsymbol{I}_{n}\right\}_{n=-\infty}^{\infty}$ and $\left\{\boldsymbol{S}_{n}\right\}_{n=-\infty}^{\infty}$ are called Full Limiting sequences of Equation (1.5).

Consider the scalar $k$-th order linear difference equation

$$
x(n+k)+p_{1}(n) x(n+k-1)+\ldots+p_{k}(n) x(n)=0 .
$$

where $k$ is a positive integer and $p_{i}: \mathbb{Z}^{+} \rightarrow \mathbb{C}$ for $i=1, \ldots, k$. Equation (1.6) is said to be of Poincarè type if the limits

$$
q_{i}=\lim _{k \rightarrow \infty} p_{i}(n), \quad i=1, \ldots, k,
$$

exist in $\mathbb{C}$. Under this hypothesis, Equation (1.6) can be regarded as a perturbation of the equation with constant coefficients

$$
x(n+k)+q_{1} x(n+k-1)+\ldots+q_{k} x(n)=0 .
$$


Theorem E [23] (Poincarè's Theorem). Suppose condition (1.7) holds. Let $\lambda_{1}, \ldots, \lambda_{k}$ be the roots of the characteristic equation

$$
\lambda^{k}+q_{1} \lambda^{k-1}+\ldots+q_{k}=0
$$

of Equation (1.8), and suppose that

$$
\left|\lambda_{i}\right| \neq\left|\lambda_{j}\right| \quad \text { for } i \neq j
$$

If $x(n)$ is a solution of (1.6) then either $x(n)=0$ for all large $n$ or there exists an index $j \in\{1, \ldots, k\}$ such that

$$
\lim _{n \rightarrow \infty} \frac{x(n+1)}{x(n)}=\lambda_{j}
$$

Set $x_{n-1}=u_{n}$ and $x_{n}=v_{n}$ in Equation (1.1) to obtain the equivalent system

$$
\begin{aligned}
& u_{n+1}=v_{n} \\
& v_{n+1}=f\left(v_{n}, u_{n}\right), \quad n=0,1, \ldots
\end{aligned}
$$

Let

$$
\boldsymbol{T}(u, v)=(v, f(v, u))
$$

The second iterate $\boldsymbol{T}^{2}$ is given by

$$
T^{2}(u, v)=(f(v, u), f(f(v, u), v))=(G(u, v), H(u, v)) .
$$

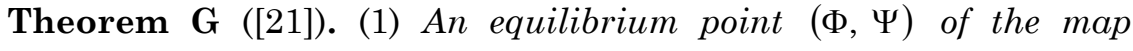
$\boldsymbol{T}^{2}=(G, H)$ is locally asymptotically stable if and only if every solution of the characteristic equation

$$
\lambda^{2}-\operatorname{tr} J_{T^{2}}(\Phi, \Psi) \lambda+\operatorname{det} J_{T^{2}}(\Phi, \Psi)=0,
$$

lies inside the unit circle, that is, if and only if

$$
\left|\operatorname{tr} J_{T^{2}}(\Phi, \Psi)\right|<1+\operatorname{det} J_{T^{2}}(\Phi, \Psi)<2 .
$$


(2) An equilibrium point $(\Phi, \Psi)$ of the map $T^{2}=(G, H)$ is locally a repeller if and only if every solution of the characteristic equation (1.12) lies outside the unit circle, that is, if and only if

$\left|\operatorname{tr} J_{T^{2}}(\Phi, \Psi)\right|<\left|1+\operatorname{det} J_{T^{2}}(\Phi, \Psi)\right| \quad$ and $\quad\left|\operatorname{det} J_{T^{2}}(\Phi, \Psi)\right|>1$.

(3) An equilibrium point $(\Phi, \Psi)$ of the map $T^{2}=(G, H)$ is locally a saddle point if the characteristic equation (1.12) has one root that lies inside the unit circle and one root that lies outside the unit circle if and only if

$$
\left|\operatorname{tr} J_{T^{2}}(\Phi, \Psi)\right|>\left|1+\operatorname{det} J_{T^{2}}(\Phi, \Psi)\right|,
$$

and

$$
\operatorname{tr} J_{T^{2}}(\Phi, \Psi)^{2}-4 \operatorname{det} J_{T^{2}}(\Phi, \Psi)>0
$$

(4) An equilibrium point $(\Phi, \Psi)$ of the map $\boldsymbol{T}^{2}=(G, H)$ is nonhyperbolic if and only if the characteristic equation (1.12) has at least one root that lies on the unit circle, that is, if and only if

$$
\left|\operatorname{tr} J_{T^{2}}(\Phi, \Psi)\right|=\left|1+\operatorname{det} J_{T^{2}}(\Phi, \Psi)\right|,
$$

or

$$
\operatorname{det} J_{T^{2}}(\Phi, \Psi)=1 \quad \text { and } \quad\left|\operatorname{tr} J_{T^{2}}(\Phi, \Psi)\right| \leq 2
$$

\section{Local Stability of the Equilibrium Points of Equation (1.1)}

In this section, we investigate the local stability character of the solutions of Equation (1.1).

The equilibrium points of Equation (1.1) are given by the relation

$$
\bar{x}=\frac{\bar{x}}{\alpha \bar{x}^{2}+\beta \bar{x}^{2}+\gamma \bar{x}} .
$$


Consequently, there are two equilibrium points of Equation (1.1): the zero equilibrium point $\bar{x}_{0}=0$ and the positive equilibrium point $\bar{x}_{+}=\left(\frac{-\gamma+\sqrt{\gamma^{2}+4(\alpha+\beta)}}{2(\alpha+\beta)}\right)$.

If we denote

$$
f(u, v)=\frac{v}{\alpha u^{2}+\beta u v+\gamma v} .
$$

Therefore, it follows that

$$
f_{u}(u, v)=\frac{-v(2 \alpha u+\beta v)}{\left(\alpha u^{2}+\beta u v+\gamma v\right)^{2}} \quad \text { and } \quad f_{v}(u, v)=\frac{\alpha u^{2}}{\left(\alpha u^{2}+\beta u v+\gamma v\right)^{2}} .
$$

Then Equation (1.1) has the linearized equation

$$
y_{n+1}=p y_{n}+q y_{n-1}
$$

whose characteristic equation is

$$
\lambda^{2}-p \lambda-q=0
$$

where

$$
p=f_{u}(\bar{x}, \bar{x})=\frac{-\bar{x}^{2}(\beta+2 \alpha)}{\left(\alpha \bar{x}^{2}+\beta \bar{x}^{2}+\gamma \bar{x}\right)^{2}} \text { and } q=f_{v}(\bar{x}, \bar{x})=\frac{\alpha \bar{x}^{2}}{\left(\alpha \bar{x}^{2}+\beta \bar{x}^{2}+\gamma \bar{x}\right)^{2}} .
$$

Proposition 1. (a) If $\gamma^{2}>\frac{4 \alpha^{2}}{\beta+3 \alpha}$, then the equilibrium point $\bar{x}_{+}$is locally asymptotically stable.

(b) If $\gamma^{2}=\frac{4 \alpha^{2}}{\beta+3 \alpha}$, then the equilibrium point $\bar{x}_{+}$is nonhyperbolic.

(c) If $\gamma^{2}<\frac{4 \alpha^{2}}{\beta+3 \alpha}$, then the equilibrium point $\bar{x}_{+}$is a saddle point. 
Proof. (I) We will prove that $|p|<1-q<2$.

(i) $|p|<1-q$

$$
\begin{aligned}
& \Leftrightarrow\left|-(\beta+2 \alpha) \bar{x}^{2}\right|<1-\alpha \bar{x}^{2} \Leftrightarrow(\beta+2 \alpha) \bar{x}^{2}<1-\alpha \bar{x}^{2} \\
& \Leftrightarrow(\beta+3 \alpha)\left(-\gamma+\sqrt{\gamma^{2}+4(\alpha+\beta)}\right)^{2}<4(\alpha+\beta)^{2} \\
& \Leftrightarrow(\beta+3 \alpha)\left[\gamma^{2}-2 \gamma \sqrt{\gamma^{2}+4(\alpha+\beta)}+\gamma^{2}+4(\alpha+\beta)\right]<4(\alpha+\beta)^{2} \\
& \Leftrightarrow(\beta+3 \alpha)\left[2 \gamma^{2}-2 \gamma \sqrt{\gamma^{2}+4(\alpha+\beta)}+4(\alpha+\beta)\right]<4(\alpha+\beta)^{2} \\
& \Leftrightarrow(\beta+3 \alpha)\left[\gamma^{2}-\gamma \sqrt{\gamma^{2}+4(\alpha+\beta)}\right]<2(\alpha+\beta)^{2}-2(\alpha+\beta)(\beta+3 \alpha) \\
& \Leftrightarrow(\beta+3 \alpha)\left[\gamma^{2}-\gamma \sqrt{\gamma^{2}+4(\alpha+\beta)}\right]<-4 \alpha(\alpha+\beta) \\
& \Leftrightarrow-\gamma(\beta+3 \alpha) \sqrt{\gamma^{2}+4(\alpha+\beta)}<-\left[4 \alpha(\alpha+\beta)+\gamma^{2}(\beta+3 \alpha)\right] \\
& \Leftrightarrow \gamma^{2}(\beta+3 \alpha)^{2}\left(\gamma^{2}+4(\alpha+\beta)\right) \\
& >16 \alpha^{2}(\alpha+\beta)^{2}+\gamma^{4}(\beta+3 \alpha)^{2}+8 \alpha \gamma^{2}(\beta+3 \alpha)(\alpha+\beta) \\
& \Leftrightarrow 4 \gamma^{2}(\beta+3 \alpha)^{2}(\alpha+\beta)>16 \alpha^{2}(\alpha+\beta)^{2}+8 \alpha \gamma^{2}(\beta+3 \alpha)(\alpha+\beta) \\
& \Leftrightarrow \gamma^{2}(\beta+3 \alpha)^{2}>4 \alpha^{2}(\alpha+\beta)+2 \alpha \gamma^{2}(\beta+3 \alpha) \\
& \Leftrightarrow \gamma^{2}(\beta+3 \alpha)[\beta+3 \alpha-2 \alpha]>4 \alpha^{2}(\alpha+\beta) \\
& \Leftrightarrow \gamma^{2}(\beta+3 \alpha)(\alpha+\beta)>4 \alpha^{2}(\alpha+\beta) \\
& \Leftrightarrow \gamma^{2}>\frac{4 \alpha^{2}}{\beta+3 \alpha},
\end{aligned}
$$

which is true by (a). 
(ii)

$$
1-q<2 \Leftrightarrow-\alpha \bar{x}^{2}<1,
$$

which is always true. Then it follows by Theorem A that $\bar{x}_{+}$is locally asymptotically stable.

(II) Observe that

$$
\begin{gathered}
|p|=|1-q| \\
\Leftrightarrow(\beta+2 \alpha) \bar{x}^{2}=\left|1-\alpha \bar{x}^{2}\right| \\
\Leftrightarrow(\beta+2 \alpha) \bar{x}^{2}=1-\alpha \bar{x}^{2} \text { or }(\beta+2 \alpha) \bar{x}^{2}=\alpha \bar{x}^{2}-1 \text { (rejected). }
\end{gathered}
$$

Now

$$
\begin{aligned}
(\beta+2 \alpha) \bar{x}^{2} & =1-\alpha \bar{x}^{2} \\
& \Leftrightarrow 4 \alpha^{2}(\beta+\alpha)+2 \alpha \gamma^{2}(3 \alpha+\beta)-\gamma^{2}(3 \alpha+\beta)^{2}=0 \\
& \Leftrightarrow \gamma^{2}=\frac{4 \alpha^{2}}{\beta+3 \alpha},
\end{aligned}
$$

which is true by (b). Then it follows by Theorem A that $\bar{x}_{+}$is nonhyperbolic stable.

(III) Now

$$
\begin{aligned}
|p| & >|1-q| \\
& \Leftrightarrow\left|-(\beta+2 \alpha) \bar{x}^{2}\right|>\left|1-\alpha \bar{x}^{2}\right| \\
& \Leftrightarrow(\beta+2 \alpha) \bar{x}^{2}>\left|1-\alpha \bar{x}^{2}\right| \\
& \Leftrightarrow-(\beta+2 \alpha) \bar{x}^{2}<1-\alpha \bar{x}^{2}<(\beta+2 \alpha) \bar{x}^{2} .
\end{aligned}
$$


First

$$
\begin{aligned}
1-\alpha \bar{x}^{2} & <(\beta+2 \alpha) \bar{x}^{2} \\
& \Leftrightarrow \beta \bar{x}^{2}+3 \alpha \bar{x}^{2}>1 \Leftrightarrow \gamma^{2}<\frac{4 \alpha^{2}}{\beta+3 \alpha},
\end{aligned}
$$

which is true by (c).

Second

$$
(\beta+2 \alpha) \bar{x}^{2}>\alpha \bar{x}^{2}-1 \Leftrightarrow(\alpha+\beta) \bar{x}^{2}>-1,
$$

which is always true. Thus the result follows by Theorem A.

\section{Permanence and Semicycles of Equation (1.1)}

In this section, we study the boundedness and semicycles of the solutions of Equation (1.1).

Theorem 1. Every solution of Equation (1.1) is bounded and persists.

Proof. Let $\left\{x_{n}\right\}_{n=-1}^{\infty}$ be a solution of Equation (1.1). It follows from Equation (1.1) that

$$
x_{n+1}=\frac{x_{n-1}}{\alpha x_{n}^{2}+\beta x_{n} x_{n-1}+\gamma x_{n-1}}<\frac{1}{\gamma}:=\sigma,
$$

then

$$
x_{n}<\sigma \text { for all } n \geq 1 \text {. }
$$

By the change of variables $x_{n}=\frac{1}{z_{n}}$ for all $n \geq 1$, Equation (1.1) can be rewritten in the form

$$
z_{n+1}=\frac{\alpha z_{n-1}+\beta z_{n}+\gamma z_{n}^{2}}{z_{n}^{2}} .
$$


Since

$$
x_{n}=\frac{1}{z_{n}} \leq \frac{1}{\gamma} \Rightarrow z_{n} \geq \gamma .
$$

Then

$$
z_{n+1}=\gamma+\frac{\alpha z_{n-1}}{z_{n}^{2}}+\frac{\beta z_{n}}{z_{n}^{2}} \leq \gamma+\frac{\alpha z_{n-1}}{\gamma^{2}}+\frac{\beta z_{n}}{\gamma^{2}}
$$

Therefore

$$
\lim _{n \rightarrow \infty} \sup z_{n} \leq \frac{\gamma}{1-\left(\frac{\alpha}{\gamma^{2}}+\frac{\beta}{\gamma^{2}}\right)}=\frac{\gamma^{3}}{\gamma^{2}-(\alpha+\beta)}
$$

and so

$$
x_{n}=\frac{1}{z_{n}}>\frac{\gamma^{2}-(\alpha+\beta)}{\gamma^{3}}:=\rho \text {. }
$$

Thus we get for some positive integer $N$ that

$$
\rho<x_{n}<\sigma \text { for all } n \geq N,
$$

so Equation (1.1) is permanent.

Theorem 2. Every solution of Equation (1.1) consists of semicycles of length one.

Proof. Assume that $\left\{x_{n}\right\}_{n=-1}^{\infty}$ be a solution of Equation (1.1) with $x_{N} \geq \bar{x}>x_{N-1}$ for some integer $N \geq n_{0} \geq 1$. As a sake of contradiction assume that $x_{N+1} \geq \bar{x}$, then it follows from Equation (1.1) that

$$
0 \leq x_{N+1}-\bar{x}=\frac{x_{N-1}}{\alpha x_{N}^{2}+\beta x_{N} x_{N-1}+\gamma x_{N-1}}-\bar{x}<\frac{\bar{x}}{(\alpha+\beta) \bar{x}^{2}+\gamma \bar{x}}-\bar{x}=0,
$$

which is a contradiction. The proof is so completed. 


\section{Global Attractor of the Equilibrium Points of Equation (1.1)}

In this section, we study the global attractor of the equilibrium point of Equation (1.1).

Theorem 3. The equilibrium point $\bar{x}$ of Equation (1.1) is global asymptotically stable if

$$
\alpha \geq \beta \quad \text { and } \quad \gamma^{2} \geq \frac{4 \alpha^{2}}{\beta+3 \alpha} .
$$

Proof. Let $\boldsymbol{P}, \boldsymbol{Q}$ be real numbers and assume that $f:[\boldsymbol{P}, \boldsymbol{Q}]^{2} \rightarrow[\boldsymbol{P}, \boldsymbol{Q}]$ is a function defined by $f(u, v)=\frac{v}{\alpha u^{2}+\beta u v+\gamma v}$, then we can easily see that the function $f(u, v)$ is non-increasing in $u$ and is non-decreasing in $v$ and it has an invariant interval $[\boldsymbol{P}, \boldsymbol{Q}]=\left[\frac{\gamma^{2}-(\alpha+\beta)}{\gamma^{3}}, \frac{1}{\gamma}\right]$.

Moreover $[\boldsymbol{P}, \boldsymbol{Q}]$ is an attracting interval, that is, $x_{n} \in[\boldsymbol{P}, \boldsymbol{Q}]$, $n \geq 1$, for every solution $\left\{x_{n}\right\}_{n=-1}^{\infty}$ of Equation (1.1).

Now suppose that $(m, M)$ is a solution of the system

$$
M=f(m, M) \quad \text { and } \quad m=f(M, m) .
$$

Then from Equation (1.1) we see that

$$
M=\frac{M}{\alpha m^{2}+\beta M m+\gamma M} \quad \text { and } \quad m=\frac{m}{\alpha M^{2}+\beta M m+\gamma m} .
$$

Then

$$
\alpha m^{2}+\beta M m+\gamma M=1, \quad \alpha M^{2}+\beta M m+\gamma m=1 .
$$

Subtracting these two equations we obtain

$$
(M-m)[\alpha(M+m)-\gamma]=0 .
$$


Now if $\alpha(M+m) \neq \gamma$, then $m=M$. If $M+m=\frac{\gamma}{\alpha}$, then it follows from (4.4) that

$$
\alpha(\alpha-\beta) m^{2}+\gamma(\beta-\alpha) m+\left(\gamma^{2}-\alpha\right)=0
$$

Equation (4.6) has no two real roots if its discriminant $\left[\gamma^{2}(\beta-\alpha)^{2}-4 \alpha\right.$ $\left.\left(\gamma^{2}-\alpha\right)(\alpha-\beta)\right]$ is non-positive, which is true if (4.1) holds. The proof follows by Theorem B.

Theorem 4. The positive equilibrium point $\bar{x}$ of Equation (1.1) is global attractor if

$$
\gamma^{2}>2 \alpha
$$

Proof. It follows by the method of full limiting sequences [12] that there exist solutions $\left\{I_{n}\right\}_{n=-\infty}^{\infty}$ and $\left\{S_{n}\right\}_{n=-\infty}^{\infty}$ of Equation (1.1) with

$$
\rho \leq I=I_{0}=\lim _{n \rightarrow \infty} \inf x_{n} \leq \lim _{n \rightarrow \infty} \sup x_{n}=S_{0}=S \leq \sigma,
$$

where

$$
I_{n}, S_{n} \in[I, S], n=0,-1, \ldots
$$

It suffices to show that $I=S$. Now it follows from Equation (1.1) that

$$
I=\frac{I_{-2}}{\alpha I_{-1}^{2}+\beta I_{-1} I_{-2}+\gamma I_{-2}} \geq f(S, I)=\frac{I}{\alpha S^{2}+\beta I S+\gamma I},
$$

and so

$$
\alpha S^{2} I+\beta S I^{2}+\gamma I^{2} \geq I \Leftrightarrow \alpha S^{2}+\beta S I+\gamma I \geq 1 .
$$

Similarly, it is easy to see from Equation (1.1) that

$$
S \leq f(I, S) .
$$


Then

$$
\alpha I^{2}+\beta S I+\gamma S \leq 1
$$

then from Equations (4.8) and (4.9) that

$$
\alpha\left(I^{2}-S^{2}\right)+\gamma(S-I) \leq 0,
$$

or

$$
(I-S)[\alpha(S+I)-\gamma] \leq 0,
$$

and so $I \geq S$ if

$$
\alpha(S+I)-\gamma \leq 0
$$

which is holding by (4.7). Thus the proof is complete.

\section{Rate of Convergence of Equation (1.1)}

In this section, we will recognize the rate of convergence of a solution that converges to the unique positive equilibrium point of Equation (1.1).

Theorem 5. Assume that $\gamma^{2}>2 \alpha$. Then all solutions of Equation (1.1) which are eventually different from the equilibrium satisfy the following:

$$
\lim _{n \rightarrow \infty} \frac{x_{n+1}-\bar{x}}{x_{n}-\bar{x}}=\frac{2\left(-(\beta+2 \alpha)+\sqrt{(\beta+2 \alpha)^{2}-\alpha\left(\gamma+\sqrt{\gamma^{2}+4(\beta+\alpha)}\right)^{2}}\right)}{\left(\gamma+\sqrt{\gamma^{2}+4(\beta+\alpha)}\right)^{2}}
$$

or

$$
\lim _{n \rightarrow \infty} \frac{x_{n+1}-\bar{x}}{x_{n}-\bar{x}}=\frac{2\left(-(\beta+2 \alpha)-\sqrt{(\beta+2 \alpha)^{2}-\alpha\left(\gamma+\sqrt{\gamma^{2}+4(\beta+\alpha)}\right)^{2}}\right)}{\left(\gamma+\sqrt{\gamma^{2}+4(\beta+\alpha)}\right)^{2}} .
$$


Proof. It follows from Equation (1.1) that

$$
\begin{aligned}
x_{n+1} & -\bar{x}=\frac{x_{n-1}}{\alpha x_{n}^{2}+\beta x_{n-1} x_{n}+\gamma x_{n-1}}-\bar{x} \\
= & \frac{x_{n-1}-\alpha x_{n}^{2} \bar{x}+\beta x_{n-1} x_{n} \bar{x}+\gamma \bar{x} x_{n-1}}{\alpha x_{n}^{2}+\beta x_{n-1} x_{n}+\gamma x_{n-1}} \\
= & \frac{\beta x_{n-1} \bar{x}^{2}+\alpha \bar{x}^{2} x_{n-1}-\alpha \bar{x}^{3}-\beta x_{n} x_{n-1} \bar{x}-\alpha x_{n}^{2} \bar{x}+\alpha x_{n} \bar{x}^{2}+\alpha \bar{x}^{3}-\alpha x_{n} \bar{x}^{2}}{\alpha x_{n}^{2}+\beta x_{n-1} x_{n}+\gamma x_{n-1}} \\
= & \frac{\alpha \bar{x}^{2}\left(x_{n-1}-\bar{x}\right)-\alpha \bar{x}^{2}\left(x_{n}-\bar{x}\right)-\beta x_{n-1} \bar{x}\left(x_{n}-\bar{x}\right)-\alpha \bar{x} x_{n}\left(x_{n}-\bar{x}\right)}{\alpha x_{n}^{2}+\beta x_{n-1} x_{n}+\gamma x_{n-1}} \\
= & \frac{-\left(\beta x_{n-1} \bar{x}+\alpha x_{n} \bar{x}+\alpha \bar{x}^{2}\right)}{\alpha x_{n}^{2}+\beta x_{n-1} x_{n}+\gamma x_{n-1}}\left(x_{n}-\bar{x}\right)+\frac{\alpha \bar{x}^{2}}{\alpha x_{n}^{2}+\beta x_{n-1} x_{n}+\gamma x_{n-1}}\left(x_{n-1}-\bar{x}\right) .
\end{aligned}
$$

Put $\theta_{n}=x_{n}-\bar{x}$. Then we obtain

$$
\theta_{n+1}+\mu_{n} \theta_{n}-\eta_{n} \theta_{n-1}=0
$$

where

$$
\mu_{n}=\frac{-\left(\beta x_{n-1} \bar{x}+\alpha x_{n} \bar{x}+\alpha \bar{x}^{2}\right)}{\alpha x_{n}^{2}+\beta x_{n-1} x_{n}+\gamma x_{n-1}},
$$

and

$$
\eta_{n}=\frac{\alpha \bar{x}^{2}}{\alpha x_{n}^{2}+\beta x_{n-1} x_{n}+\gamma x_{n-1}} .
$$

As the positive equilibrium is a global attractor by Theorem $\mathrm{E}$, we get

$$
\lim _{n \rightarrow \infty} \mu_{n}=\frac{-\bar{x}(\beta+2 \alpha)}{((\alpha+\beta) \bar{x}+\gamma)}=-\bar{x}^{2}(\beta+2 \alpha),
$$

and

$$
\lim _{n \rightarrow \infty} \eta_{n}=\frac{\alpha \bar{x}}{((\alpha+\beta) \bar{x}+\gamma)}=\alpha \bar{x}^{2}
$$

Thus the limiting equation of (1.1) is the linearized equation (2.2). 


\section{Periodic Solutions}

In this section, we present results for the existence of minimal periodtwo solutions of Equation (1.1).

Theorem 6. (a) Equation (1.1) has minimal period-two solutions of the form $\left\{\ldots, \frac{1}{\gamma}, 0, \frac{1}{\gamma}, 0, \ldots\right\}$.

(b) Equation (1.1) has positive periodic solutions of prime period-two if and only if

$$
\beta<\alpha \quad \text { and } \quad \gamma^{2}<\frac{4 \alpha^{2}}{\beta+3 \alpha}
$$

Proof. (a) Assume that $\left\{x_{n}\right\}_{n=-1}^{\infty}$ be a solution of Equation (1.1) with $x_{-1}=0, x_{0}=\frac{1}{\gamma}\left(\right.$ or $\left.x_{-1}=\frac{1}{\gamma}, x_{0}=0\right)$, it follows by directed substitutions from Equation (1.1) that

$$
\begin{gathered}
x_{1}=0, \quad x_{2}=\frac{x_{0}}{\alpha x_{1}^{2}+\beta x_{1} x_{0}+\gamma x_{0}}=\frac{1}{\gamma}, \\
x_{3}=\frac{x_{1}}{\alpha x_{2}^{2}+\beta x_{2} x_{1}+\gamma x_{1}}=0, \\
x_{4}=\frac{x_{2}}{\alpha x_{3}^{2}+\beta x_{3} x_{2}+\gamma x_{2}}=\frac{1}{\gamma} .
\end{gathered}
$$

By continuing in this way, similarly it is easy to obtain that

$$
x_{2 n+1}=0 \text { and } x_{2 n}=\frac{1}{\gamma} .
$$

Then $\left\{0, \frac{1}{\gamma}, 0, \frac{1}{\gamma}, \ldots\right\}$ be a two cycle solution of Equation (1.1). 
(b) First suppose that there exists a minimal period-two solution $\{\ldots, \phi, \psi, \phi, \psi, \ldots\}$ of Equation (1.1), where $\phi$ and $\psi$ are distinct positive real numbers. Then $\phi, \psi$ satisfy the following:

$$
\phi=\frac{\phi}{\beta \psi \phi+\alpha \psi^{2}+\gamma \phi},
$$

and

$$
\psi=\frac{\psi}{\beta \psi \phi+\alpha \phi^{2}+\gamma \psi},
$$

which is equivalent to

$$
\beta \psi \phi+\alpha \psi^{2}+\gamma \phi-1=0,
$$

and

$$
\beta \psi \phi+\alpha \phi^{2}+\gamma \psi-1=0 .
$$

Subtracting (6.3) from (6.2) we have

$$
\alpha\left(\psi^{2}-\phi^{2}\right)+\gamma(\phi-\psi)=0 .
$$

Since $\phi \neq \psi$, we have that

$$
\phi+\psi=\gamma / \alpha .
$$

Substituting (6.4) in (6.3) we obtain

$$
\phi \psi=\frac{\alpha-\gamma^{2}}{\alpha(\beta-\alpha)},
$$

from which

$$
\phi_{ \pm}=\frac{1}{2 \alpha}\left(\gamma \pm \sqrt{\gamma^{2}-\frac{4 \alpha\left(\alpha-\gamma^{2}\right)}{(\beta-\alpha)}}\right) .
$$


Equation (6.4) implies that

$$
\psi_{ \pm}=\frac{\gamma}{\alpha}-\phi_{ \pm}=\phi_{\mp} .
$$

Since $\psi_{ \pm}=\phi_{\mp}$ are distinct real numbers, $\gamma^{2}(\beta-\alpha)^{2}-4 \alpha\left(\alpha-\gamma^{2}\right)(\beta-\alpha)>0$, which implies that $\frac{4 \alpha^{2}}{\beta+3 \alpha}>\gamma^{2}$ and $\alpha>\beta$. Thus Equation (6.1) holds.

Second suppose that the condition (6.1) is true. We will show that Equation (1.1) has positive prime period two solutions.

Now choose

$$
x_{-1}=\psi=\frac{1}{2 \alpha}\left[\gamma+\sqrt{\gamma^{2}-4 \alpha\left(\alpha-\gamma^{2}\right) /(\beta-\alpha)}\right],
$$

and

$$
x_{0}=\phi=\frac{1}{2 \alpha}\left[\gamma-\sqrt{\gamma^{2}-4 \alpha\left(\alpha-\gamma^{2}\right) /(\beta-\alpha)}\right] .
$$

It is easy to prove that

$$
x_{1}=x_{-1} \quad \text { and } \quad x_{2}=x_{0} .
$$

Then it follows by induction that

$$
x_{2 n}=\phi \text { and } x_{2 n+1}=\psi \text { for all } n \geq-1 .
$$

Thus Equation (1.1) has the positive prime period two solution

$$
\ldots, \phi, \psi, \phi, \psi, \ldots,
$$

where $\phi$ and $\psi$ are the distinct roots of the quadratic equation (6.6) and the proof is completed.

\section{Local Stability Analysis of the Period two Solutions}

Theorem 7. The minimal period-two solutions is as follows:

(i) The minimal period-two solution $\left\{\ldots, \frac{1}{\gamma}, 0, \frac{1}{\gamma}, 0, \ldots\right\}$ is locally asymptotically stable if $\alpha<\gamma^{2}$. 
(ii) The minimal period-two solution $\left\{\ldots, \frac{1}{\gamma}, 0, \frac{1}{\gamma}, 0, \ldots\right\}$ is a nonhyperbolic if $\alpha=\gamma^{2}$.

(iii) The minimal period-two solution $\left\{\ldots, \frac{1}{\gamma}, 0, \frac{1}{\gamma}, 0, \ldots\right\}$ is a saddle point if $\alpha>\gamma^{2}$.

(iv) If $\beta<\alpha$ and $\frac{4 \alpha^{2}}{\beta+3 \alpha}>\gamma^{2}$, then the minimal period-two solution $\{\ldots, \phi, \psi, \phi, \psi, \ldots\}$ is a saddle point.

Proof. By substitution $x_{n-1}=u_{n}, x_{n}=v_{n}$ Equation (1.1) becomes the system of equations

$$
\left\{\begin{array}{c}
u_{n+1}=v_{n}, \\
v_{n+1}=\frac{u_{n}}{\beta v_{n} u_{n}+\alpha v_{n}^{2}+\gamma u_{n}} .
\end{array}\right.
$$

The map $T$ corresponding to (7.1) is of the form

$$
T\left(\begin{array}{l}
v \\
u
\end{array}\right)=\left(\begin{array}{c}
v \\
h(u, v)
\end{array}\right)
$$

where

$$
h(u, v)=\frac{u}{\beta v u+\alpha v^{2}+\gamma u} .
$$

Now the second iteration of the map $T$ is

$$
T^{2}\left(\begin{array}{l}
v \\
u
\end{array}\right)=T\left(\begin{array}{c}
v \\
h(u, v)
\end{array}\right)=\left(\begin{array}{c}
h(u, v) \\
h(v, h(u, v))
\end{array}\right)=\left(\begin{array}{c}
G(u, v) \\
H(u, v)
\end{array}\right),
$$

where

$$
H(u, v)=\frac{v}{\beta v h(u, v)+\alpha h(u, v)^{2}+\gamma u}
$$


and

$$
G(u, v)=\frac{u}{\beta v u+\alpha v^{2}+\gamma u}
$$

Let $\left(\begin{array}{l}\phi \\ \psi\end{array}\right)$ be the fixed point to $T^{2}$. Then the Jacobian matrix $J_{T^{2}}$ is given by

$$
J_{T^{2}}(\phi, \psi)=\left(\begin{array}{ll}
\frac{\partial G(\phi, \psi)}{\partial u} & \frac{\partial G(\phi, \psi)}{\partial v} \\
\frac{\partial H(\phi, \psi)}{\partial u} & \frac{\partial H(\phi, \psi)}{\partial v}
\end{array}\right)
$$

where

$$
\begin{aligned}
\frac{\partial G(\phi, \psi)}{\partial u} & =\frac{\left(\beta \phi \psi+\alpha \psi^{2}+\gamma \phi\right)-\phi(\beta \psi+\gamma)}{\left(\beta \phi \psi+\alpha \psi^{2}+\gamma \phi\right)^{2}}=\alpha \psi^{2}, \\
\frac{\partial G(\phi, \psi)}{\partial v} & =\frac{-\phi(\beta \phi+2 \alpha \psi)}{\left(\beta \phi \psi+\alpha \psi^{2}+\gamma \phi\right)^{2}}=-\left(\beta \phi^{2}+2 \alpha \phi \psi\right), \\
\frac{\partial H(\phi, \psi)}{\partial u} & =\frac{-\psi\left(\beta h(\phi, \psi)+2 \alpha\left(h(\phi, \psi) \frac{\partial h}{\partial u}(\phi, \psi)\right)\right)}{\left(\beta h(\phi, \psi) \psi+\alpha h(\phi, \psi)^{2}+\gamma \psi\right)^{2}}, \\
& =-\left(\alpha \beta \psi^{4}+2 \alpha^{2} \phi \psi^{3}\right),
\end{aligned}
$$

and

$$
\begin{aligned}
\frac{\partial H(\phi, \psi)}{\partial v}= & \frac{\left(\beta h(\phi, \psi) \psi+\alpha h(\phi, \psi)^{2}+\gamma \psi\right)}{\left(\beta h(\phi, \psi) \psi+\alpha h(\phi, \psi)^{2}+\gamma \psi\right)^{2}} \\
& -\frac{\psi\left(\beta h(\phi, \psi)+\beta \psi \frac{\partial h}{\partial v}(\phi, \psi)+2 \alpha\left(h(\phi, \psi) \frac{\partial h}{\partial v}(\phi, \psi)\right)+\gamma\right)}{\left(\beta h(\phi, \psi) \psi+\alpha h(\phi, \psi)^{2}+\gamma \psi\right)^{2}} \\
= & \alpha \phi^{2}+\beta^{2} \psi^{2} \phi^{2}+2 \alpha \beta \phi \psi^{3}+2 \alpha \beta \phi^{3} \psi+4 \alpha^{2} \phi^{2} \psi^{2} .
\end{aligned}
$$


(i) The Jacobian matrix of the map $T^{2}$ at the points $p_{x}$ and $p_{y}$ is of the form

$$
J_{T^{2}}\left(p_{x}\right)=\left(\begin{array}{ll}
0 & \frac{-\beta}{\gamma^{2}} \\
0 & \frac{\alpha}{\gamma^{2}}
\end{array}\right), J_{T^{2}}\left(p_{y}\right)=\left(\begin{array}{cc}
\frac{\alpha}{\gamma^{2}} & 0 \\
\frac{-\beta \alpha}{\gamma^{2}} & 0
\end{array}\right)
$$

with the eigenvalues $\lambda_{1}=0$ and $\lambda_{2}=\frac{\alpha}{\gamma^{2}}$ which means by Theorem G that the periodic solution $\left\{\ldots, \frac{1}{\gamma}, 0, \frac{1}{\gamma}, 0, \ldots\right\}$ is locally asymptotically stable if $\alpha<\gamma^{2}$,

(ii) If $\alpha=\gamma^{2}$, then the minimal period-two solution $\left\{\ldots, \frac{1}{\gamma}, 0, \frac{1}{\gamma}, 0, \ldots\right\}$ is a non-hyperbolic point.

(iii) If $\alpha>\gamma^{2}$, then the minimal period-two solution $\left\{\ldots, \frac{1}{\gamma}, 0, \frac{1}{\gamma}, 0, \ldots\right\}$ is a saddle point.

(iv) The Jacobian matrix of the map $T^{2}$ at the point $\{\ldots, \phi, \phi, \phi, \phi, \ldots\}$ using (7.2)-(7.5) is of the form

$$
J_{T^{2}}(\phi, \psi)=\left(\begin{array}{cc}
\alpha \psi^{2} & -\left(\beta \phi^{2}+2 \alpha \phi \psi\right) \\
-\left(\alpha \beta \psi^{4}+2 \alpha^{2} \phi \psi^{3}\right) & \alpha \phi^{2}+\left(4 \alpha^{2}+\beta^{2}\right) \psi^{2} \phi^{2}+2 \alpha \beta \phi \psi\left(\phi^{2}+\psi^{2}\right)
\end{array}\right) .
$$

Now by (6.4) and (6.5), we have

$$
p=\operatorname{tr} J_{T^{2}}(\phi, \psi)=\alpha\left(\phi^{2}+\psi^{2}\right)+\left(4 \alpha^{2}+\beta^{2}\right) \psi^{2} \phi^{2}+2 \alpha \beta \phi \psi\left(\phi^{2}+\psi^{2}\right),
$$

and

$$
q=\operatorname{Det}_{T^{2}}(\phi, \psi)=(\alpha \phi \psi)^{2}
$$


We have that $(\phi, \psi)$ is a saddle point if

$$
\begin{aligned}
|p|>1+q & \\
\Leftrightarrow & \alpha\left(\phi^{2}+\psi^{2}\right)+\left(3 \alpha^{2}+\beta^{2}\right) \psi^{2} \phi^{2}+2 \alpha \beta \phi \psi\left(\phi^{2}+\psi^{2}\right)>1 \\
\Leftrightarrow & \left(\alpha-\gamma^{2}\right)\left(\left(3 \alpha^{2}+\beta^{2}\right)\left(\alpha-\gamma^{2}\right)-2 \alpha^{2}(\beta-\alpha)+2 \beta \gamma^{2}(\beta-\alpha)-4 \alpha \beta\left(\alpha-\gamma^{2}\right)\right) \\
& >\alpha(\beta-\alpha)^{2}\left(\alpha-\gamma^{2}\right) \\
\Leftrightarrow & (\beta-\alpha)\left((\beta-3 \alpha)\left(\alpha-\gamma^{2}\right)-2 \alpha^{2}+2 \beta \gamma^{2}-\alpha(\beta-\alpha)\right)>0 .
\end{aligned}
$$

This completes the proof.

\section{Numerical Examples}

To confirm the results of this paper, we consider numerical examples which rep-resent different types of solutions to Equation (1.1).

Example 1. We assume $x_{-1}=0.4, x_{0}=0.3, \alpha=0.4, \beta=5, \gamma=0.5$ (see Figure 1).

Example 2. (See Figure 2), since $x_{-1}=0.003, x_{0}=0.5, \alpha=30$, $\beta=0.5, \gamma=18$.

Example 3. We assume $x_{-1}=2, x_{0}=3, \alpha=5, \beta=1.5, \gamma=0.04$ (see Figure 3).

Example 4. (See Figure 4), since $x_{-1}=0.9, x_{0}=3, \alpha=0.025$, $\beta=10, \gamma=0.125$.

Example 5. We consider $x_{-1}=0.3, x_{0}=0.2, \alpha=13, \beta=9, \gamma=3.5$ (see Figure 5).

Example 6. (See Figure 6), since $x_{-1}=2, x_{0}=3, \alpha=4, \beta=6$, $\gamma=1.5$. 
GLOBAL DYNAMICS OF SOME FRACTIONAL ...

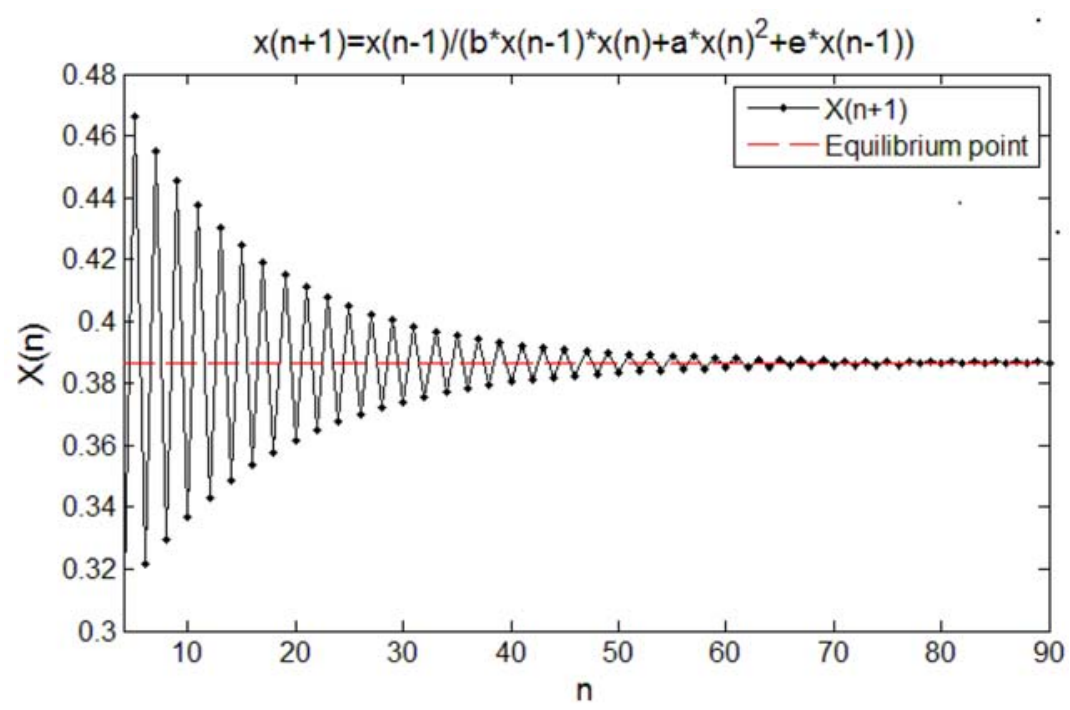

Figure 1.

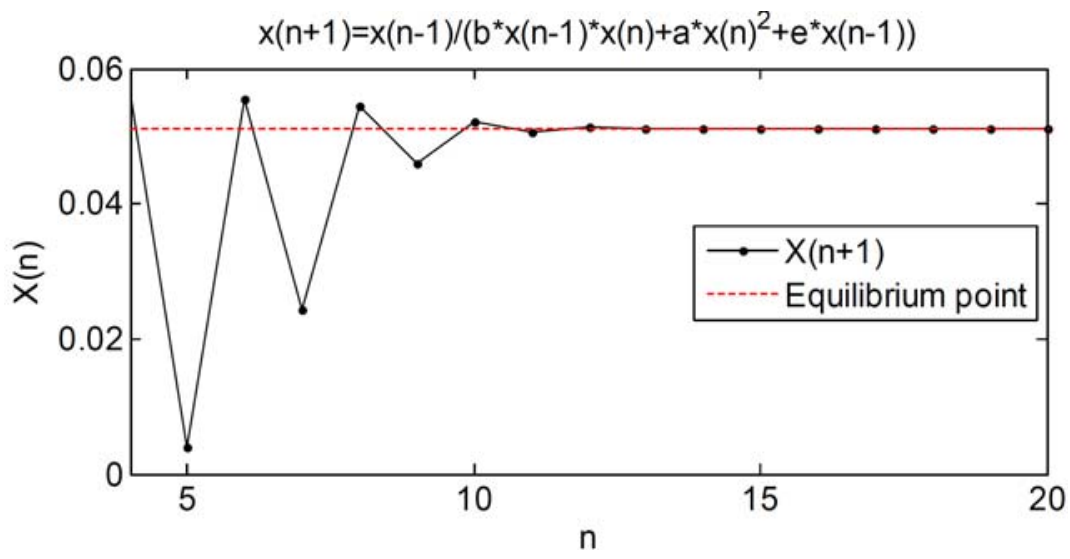

Figure 2. 


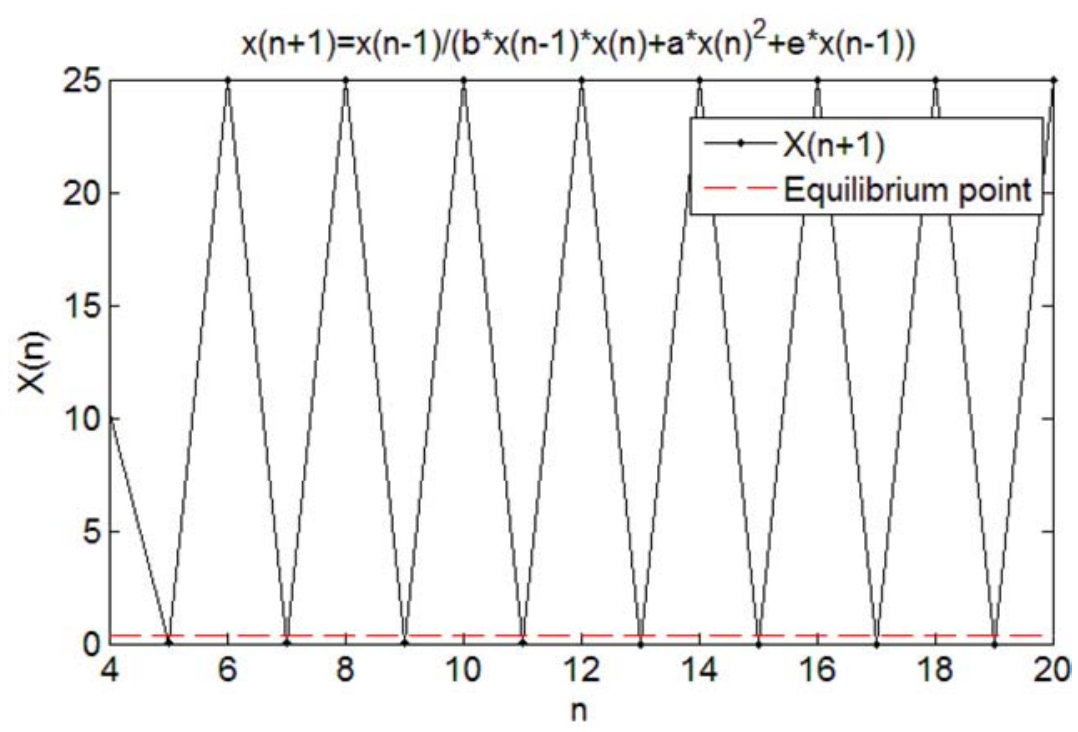

Figure 3.

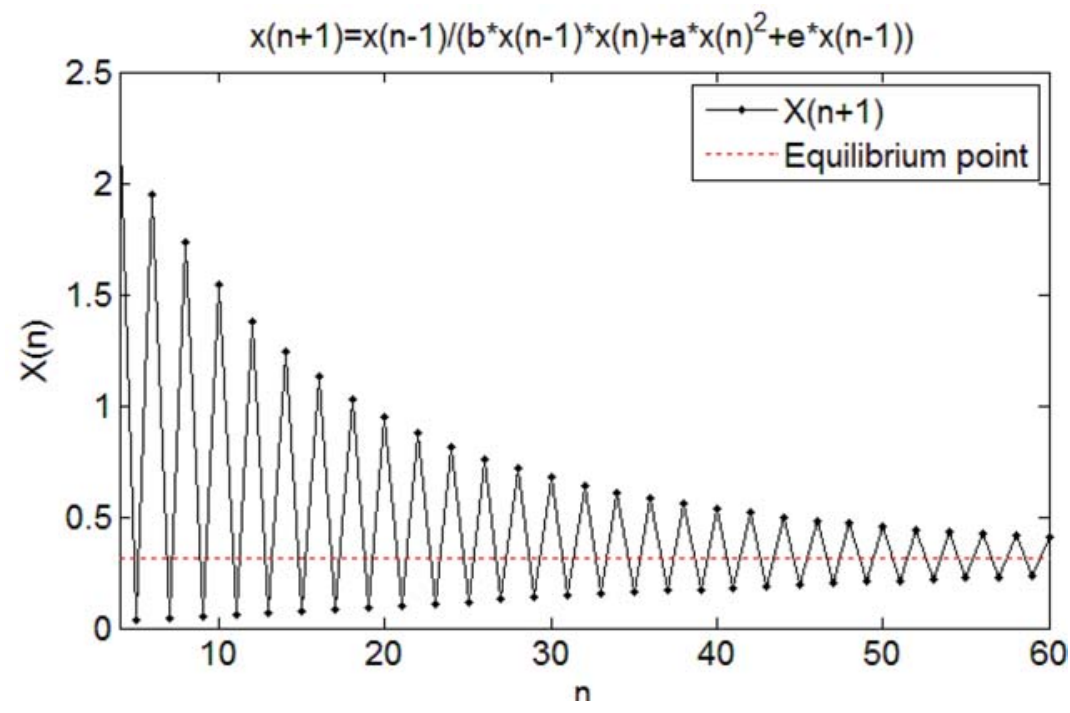

Figure 4. 


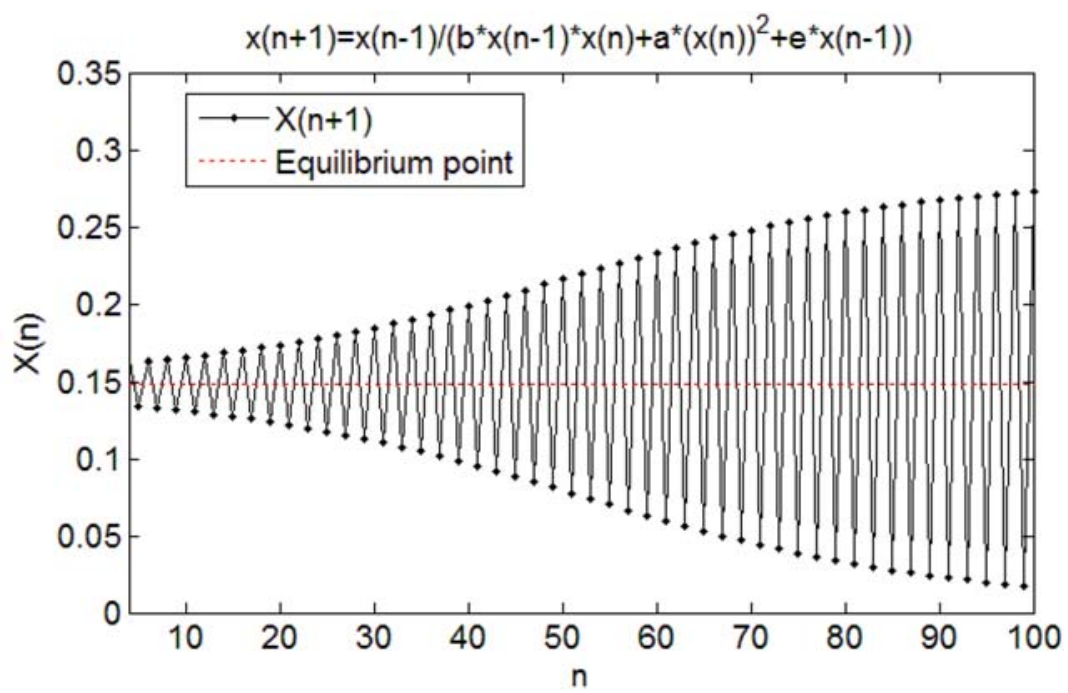

Figure 5.

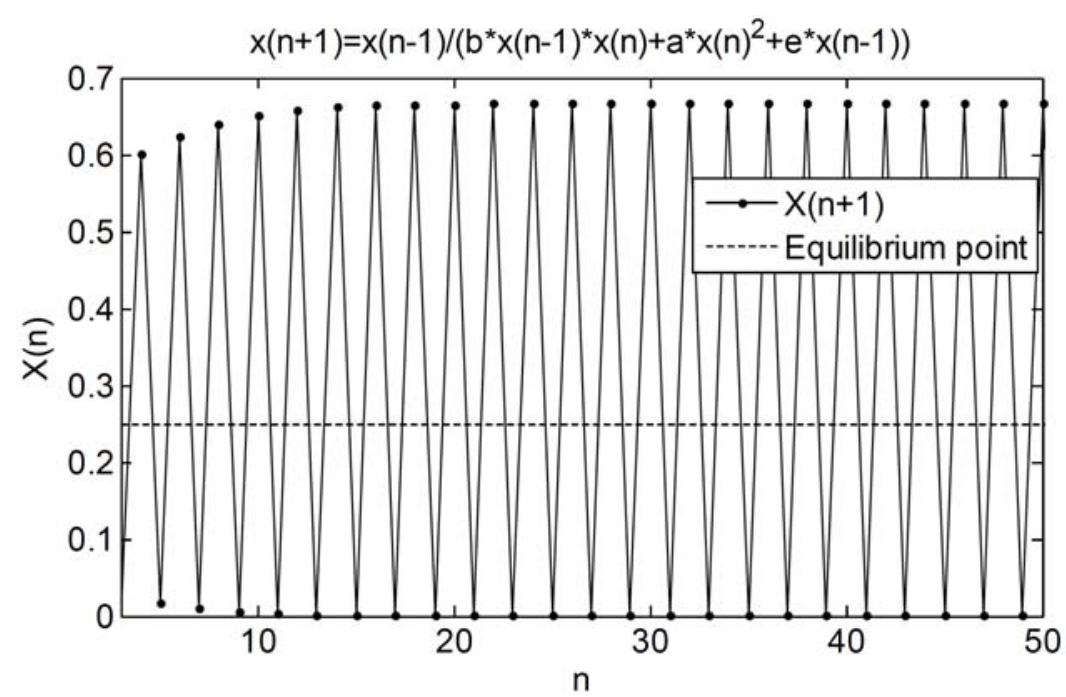

Figure 6. 


\section{References}

[1] E. Camouzis and G. Ladas, Dynamics of Third-Order Rational Difference Equations with Open Problems and Conjectures, Chapman and Hall/CRC, 2007.

[2] E. Camouzis and G. Ladas, When does local asymptotic stability imply global attractivity in rational equations?, Journal of Difference Equations and Applications $12(8)$ (2006), 863-885.

DOI: https://doi.org/10.1080/10236190600772663

[3] M. Dipippo, Global Dynamics of Some Quadratic Difference Equations, University of Rhode Island, 2016.

[4] H. El-Metwally, E. A. Grove and G. Ladas, A global convergence result with applications to periodic solutions, Journal of Mathematical Analysis and Applications 245(1) (2000), 161-170.

DOI: https://doi.org/10.1006/jmaa.2000.6747

[5] E. M. Elabbasy, H. El-Metwally and E. M. Elsayed, Global attractivity and periodic character of a fractional difference equation of order three, Yokohama Mathematical Journal 53 (2007), 89-100.

[6] H. El-Metwally, Qualitative properties of some higher order difference equations, Computers \& Mathematics with Applications 58(4) (2009), 686-692.

DOI: https://doi.org/10.1016/j.camwa.2009.03.092

[7] H. El-Metwally and E. M. Elsayed, Qualitative behavior of some rational difference equations, Journal of Computational Analysis and Applications 20(2) (2016), 226-236.

[8] E. M. Elsayed and H. El-Metwally, Global behavior and periodicity of some difference equations, Journal of Computational Analysis and Applications 19(2) (2015), 298-309.

[9] H. El-Metwally, I. Yalcinkaya and C. Cinar, Global stability of an economic model, Utilitas Mathematica 95 (2014), 235-244.

[10] M. Garić-Demirović, M. R. S. Kulenović and M. Nurkanović, Global dynamics of certain homogeneous second-order quadratic fractional difference equation, The Scientific World Journal (2013), Article ID 210846, pages 1-10.

DOI: http://dx.doi.org/10.1155/2013/210846

[11] M. Garić-Demirović, M. R. S. Kulenović and M. Nurkanović, Basins of attraction of equilibrium points of second order difference equations, Applied Mathematics Letters 25(12) (2012), 2110-2115.

DOI: https://doi.org/10.1016/j.aml.2012.05.009

[12] C. Gibbons, M. R. S. Kulenović and G. Ladas, On the recursive sequence $x_{n+1}=\left(\alpha+\beta x_{n-1}\right) /\left(\gamma+x_{n}\right)$, Mathematical Sciences Research Hotline 4(2) (2000), $1-11$. 
[13] S. J. Hrustić, M. R. S. Kulenović and M. Nurkanović, Global dynamics and bifurcations of certain second order rational difference equation with quadratic terms, Qualitative Theory of Dynamical Systems 15(1) (2016), 283-307.

DOI: https://doi.org/10.1007/s12346-015-0148-x

[14] S. Jašarević and M. R. S. Kulenović, Basins of attraction of equilibrium and boundary points of second-order difference equations, Journal of Difference Equations and Applications 20(5-6) (2014), 947-959.

DOI: https://doi.org/10.1080/10236198.2013.855733

[15] S. Kalabušic, M. R. S. Kulenović and E. Pilav, Global dynamics of anti-competitive systems in the plane, Dynamics of Continuous, Discrete and Impulsive Systems Series A: Mathematical Analysis 20(4) (2013), 477-505.

[16] M. R. S. Kulenović and G. Ladas, Dynamics of second order rational difference equations: With open problems and conjectures, Chapman and Hall/CRC, 2001.

[17] M. R. S. Kulenović, S. Moranjkic and Z. Nurkanovic, Naimark-Sacker bifurcation of second order rational difference equation with quadratic terms, Journal of Nonlinear Sciences and Applications 10(7) (2017), 3477-3489.

DOI: http://dx.doi.org/10.22436/jnsa.010.07.11

[18] M. R. S. Kulenović, and O. Merino, Global bifurcation for discrete competitive systems in the plane, Discrete \& Continuous Dynamical Systems-B 12(1) (2009), 133-149.

DOI: http://dx.doi.org/10.3934/dcdsb.2009.12.133

[19] M. R. S. Kulenović, and O. Merino, Invariant manifolds for competitive discrete systems in the plane, International Journal of Bifurcation and Chaos 20(8) (2010), 2471-2486.

DOI: https://doi.org/10.1142/S0218127410027118

[20] M. R. S. Kulenović, S. Moranjkić and Z. Nurkanović, Global dynamics and bifurcation of a perturbed Sigmoid Beverton-Holt difference equation, Mathematical Methods in the Applied Sciences 39(10) (2016), 2696-2715.

DOI: https://doi.org/10.1002/mma.3722

[21] M. R. S. Kulenović and O. Merino, Discrete Dynamical Systems and Difference Equations with Mathematica, CRC Press, 2002.

[22] G. L. Karakostas, A discrete semi-flow in the space of sequences and study of convergence of sequences defined by difference equations, M. E. Greek Math. Soc. 30 (1989), 66-74.

[23] M. Pituk, More on Poincaré's and Perron's Theorems for Difference Equations, Journal of Difference Equations and Applications 8(3) (2002), 201-216.

DOI: https://doi.org/10.1080/10236190211954 